\title{
A new species of fruit bat (Megachiroptera: Pteropodidae: Thoopterus) from Sulawesi and adjacent islands, Indonesia
}

\author{
Ibnu Maryanto', Mohamad Yani², Siti Nuramaliati Prijono' and Sigit Wiantoro' \\ ${ }^{1}$ Museum Zoologicum Bogoriense, Research Center for Biology-LIPI, JI. Jakarta-Bogor Km 46, \\ Cibinong 16911, Indonesia. Email: ibnu_mar@yahoo.com \\ 2 Jl. Gotong Royong, Gang Jeruk 6/2, Komplek Kebon Jeruk, Pejeruk, Ampenan, \\ Mataram Lombok, Indonesia.
}

\begin{abstract}
A new species of fruit bat in the genus Thoopterus, T. suhaniahae, is described from Sulawesi, Talaud and Wowoni Islands in Indonesia. The new species differs from T. nigrescens in having larger cranial, dental, mandibular, external measurements, and a wider calcar. In addition, the tail of the new species is absent or reduced to a rudiment, a quarter to a half of the under face of the uropatagium is sparsely furred and the urethal aperture of the glans penis lacks a conspicuous scale-like projection. The new species is sympatric with $T$. nigrescens and is known from elevations of $60-2,100 \mathrm{~m}$ above sea level.
\end{abstract}

KEYWORDS: taxonomy, morphology, Thoopterus suhaniahae sp. nov.

\section{INTRODUCTION}

The fruit bat genus Thoopterus was proposed by Matschie (1899) as a subgenus of Cynopterus. Gray (1870) recognized nigrescens as a variety of Cynopterus marginatus (Geoffroy, 1810). Andersen (1912) recognized Thoopterus as a valid monotypic genus, based on the absence of a postorbital foramina, incisors complete, upper canine grooved, cheek teeth $4 / 5, \mathrm{P}_{4}$ and $\mathrm{M}_{1}$ broad, quadrate with large surface, tail reduced to a rudiment, membranes attached on the second toe, fur much longer compared to Cynopterus, and grooved upper canines without cingulum cusps.

Thoopterus nigrescens has been recorded from Morotai in the North Moluccas (the type locality) and Sulawesi. Bergmans and Rozendaal (1988) and Suyanto et al. (1998) reported it occurring on the Sangihe Talaud Islands. Boeadi and Saim collected this species from Sula and Kabaena, Buton in 1991 and 2002, and from Wowoni Islands in 2005 which were then deposited in the Museum Zoologicum Bogoriense (MZB). Bergmans and Rozendaal (1988) reported that specimens from South-West Sulawesi (Bantimurung and Malino) were larger than that from northern Sulawesi and may represent a new taxon. Thoopterus nigrescens has previously been reported from sea level up to 1,780 m (Bergmans and Rozendaal 1988). During a 2000-2001 survey, however, we found $T$. nigrescens to be most common at even higher altitudes of Nokilalaki and Rorekatimbu-Lore Lindu National Park: 2,100 $\mathrm{m}$ and 2,200 m, respectively
(Maryanto and Yani 2001). To clarify the taxonomic status of these populations, this paper provides a morphological description of these high elevation populations of Thoopterus from Sulawesi and adjacent islands. We found compelling evidence to describe these populations as a new species of Thoopterus, here named T. suhaniahae sp. nov.

\section{MATERIALS AND METHODS}

A total of 102 adult specimens of Thoopterus from Sulawesi, Buton, Sula, Talaud and Wowoni Islands were included in our morphometric analyses (see specimens examined and Figure 1). All cranial, dental, mandibular and external measurements are given in $\mathrm{mm}$. Measurements used in this paper follow Kitchener et al. (1995) and Kitchener and Maharadatunkamsi (1991) and are as follows:

Skull and dentition: greatest skull length (GSL), interorbital breadth (IO), zygomatic breadth (ZB), rostrum length (RL), mastoid breadth $(\mathrm{MB})$, braincase height (HB), mesopterygoid fossa width (MSF), palatal length (LOP), bulla length (BL), postorbital width (POW), braincase width (BW), dentary length (DL), height of ramus at angular process (RAP), $\mathrm{C}^{1}-\mathrm{C}^{1}$ breadth (outside), $\mathrm{M}^{1}-\mathrm{M}^{1}, \mathrm{M}^{2}-\mathrm{M}^{2}, \mathrm{P}^{3}-\mathrm{P}^{3}, \mathrm{P}^{4}-\mathrm{P}^{4}$ (alveoli, inside), $\mathrm{C}^{1}-\mathrm{M}^{2}$ (crown length), $\mathrm{C}_{1}-\mathrm{M}_{2}$ (crown length), $\mathrm{M}^{1}$ crown length $\left(\mathrm{M}^{1} \mathrm{~L}\right), \mathrm{M}^{1}$ crown width $\left(\mathrm{M}^{1} \mathrm{~W}\right), \mathrm{M}^{2}$ crown length $\left(\mathrm{M}^{2} \mathrm{~L}\right), \mathrm{M}^{2}$ crown width $\left(\mathrm{M}^{2} \mathrm{~W}\right), \mathrm{P}^{3}$ crown length $\left(\mathrm{P}^{3} \mathrm{~L}\right), \mathrm{P}^{3}$ crown width $\left(\mathrm{P}^{3} \mathrm{~W}\right), \mathrm{P}^{4}$ crown length $\left(\mathrm{P}^{4} \mathrm{~L}\right), \mathrm{P}^{4}$ 
crown width $\left(\mathrm{P}^{4} \mathrm{~W}\right)$.

External: forearm length (FA), head and body length (HBL), tail length (TAIL), ear length (EAR), tibia length (TIBIA), metacarpal 2 length (P2), metacarpal 2 phalanx 1 length (P2_1), metacarpal 3 length (P3), metacarpal 3 phalanx 1 length (P3 1), digit 4 phalanx 1 length (P4_1), metacarpal 5 length (P5), metacarpal 5 phalanx 1 length (P5_1). Adults were identified as those specimens with basioccipital and basisphenoid bones completely fused and epiphyseal swelling of metacarpal joints absent. Sexual dimorphism in skull and external characters was analyzed by a 2-way ANOVA. We tested for sexual dimorphism and morphological characters using canonical discriminant function analyses (DFA) of Thoopterus groups. Multiple analysis of variance (MANOVA) was run separately for craniodental and external characters. Colour terminology follows Kornerup and Wanscher (1984). Specimens discussed here are deposited in the Natural History Museum, London (BMNH) and the Museum Zoologicum Bogoriense, Cibinong Bogor (MZB).

\section{RESULTS}

\section{UNIVARIATE STATISTICS}

\section{Skull, dentary, dentition}

Thoopterus suhaniahae sp. nov. males are larger in most dimensions than females; respective measurements for males and females are as follows:
GSL 38.85 (37.21-40.55) v. 37.10 (35.82-38.28); ZB 25.12 (23.76-26.35) v. 23.93 (22.10-25.23); HB 12.77 (10.41-14.60) v. 12.78 (11.34-14.19); RL 9.54 (8.95$10.75)$ v. 9.20 (8.54-10.01); IO 8.71 (7.89-9.34) v. 8.24 (7.51-8.58); $\mathrm{C}^{1-}-\mathrm{C}^{1} 8.06$ (7.57-8.58) v. 7.68 (7.23-7.83); POW 7.62 (7.17-8.26) v. 7.51 (7.20-7.91); LOP 19.60 (18.37-20.92) v. 18.84 (18.10-20.03); MSF 4.59 (4.16$5.03)$ v. 4.64 (4.23-5.42); BL 3.03 (2.20-3.68) v. 3.00 (2.42-3.53); RAP 15.32 (13.63-17.01) v. 14.11 (13.1214.95); DL 29.33 (28.03-31.06) v. 27.67 (25.58-28.91) (Table 1).

Cranial, dental, mandibular and external dimensions in $T$. suhaniahae sp. nov. are mostly larger than $T$. nigrescens. For example, measurements of female $T$. suhaniahae sp. nov. and T. nigrescens are respectively (mean, range in mm): GSL 37.10 (35.82-38.28) v. 34.29 (33.06-35.62), RL 9.20 (8.54-10.01) v. 8.39 (7.57-9.14), ZB relatively wide $23.93(22.1-25.23)$ v. 21.67 (20.0223.31), HB 12.78 (11.34-14.19) v. 11.65 (11.14-12.04); for males, the equivalent comparisons are GSL 38.85 (37.21-40.55) v. 35.33 (32.55-37.67), RL 9.54 (8.95$10.75)$ v. 8.65 (7.22-9.61), ZB 25.12 (23.76.1-26.35) v. 22.125 (19.39-23.71), HB 12.77 (10.41-14.60) v. 11.81 (10.88-12.50) (Table 1).

\section{External features}

In T. suhaniahae sp. nov., external measurements are larger in males than females, for example, HBL (in mm) 98.47 (89.35-109.67) v. 94.91 (86.02-101.11); ear length $17.79(16.62-19.06)$ v. $17.64(16.62-19.50)$; FA 78.36 (74.42-81.77) v. 76.44 (73.03-78.47); Tibia 32.26

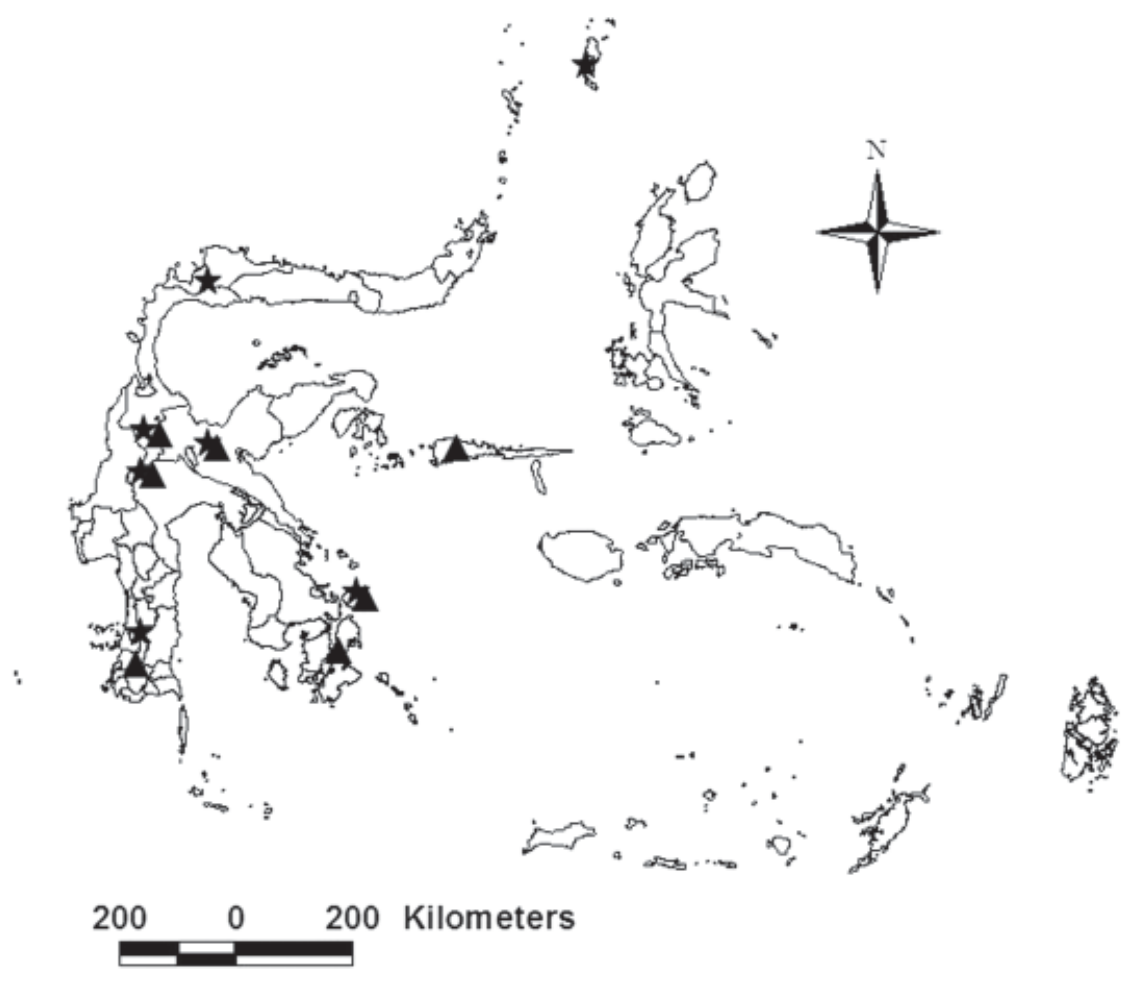

FIGURE 1 Collection localities of Thoopterus spp. used in this study, T. nigrescens ( $\mathbf{\Delta})$ and T. suhaniahae sp. nov. ( $\star$ ). 
TABLE 1 Measurements $(\mathrm{mm})$ for skull includes dentary, dental and external characters (see Material and Methods section for explanation of character codes) of adult Thoopterus spp.

\begin{tabular}{|c|c|c|c|c|c|}
\hline q Thoopterus nigrescens & Mean & $\begin{array}{l}\text { Standard } \\
\text { deviation }\end{array}$ & Minimum & Maximum & $\mathrm{N}$ \\
\hline GSL & 34.29 & 0.69 & 33.06 & 35.62 & 31 \\
\hline ZB & 21.67 & 0.73 & 20.02 & 23.31 & 31 \\
\hline HB & 11.66 & 0.26 & 11.14 & 12.04 & 31 \\
\hline RL & 8.39 & 0.37 & 7.57 & 9.14 & 31 \\
\hline IO & 7.37 & 0.31 & 6.58 & 7.99 & 31 \\
\hline $\mathrm{C}^{1}-\mathrm{C}^{1}$ & 6.76 & 0.22 & 6.3 & 7.17 & 31 \\
\hline POW & 6.83 & 0.28 & 6.13 & 7.33 & 31 \\
\hline LOP & 7.23 & 0.5 & 16.19 & 18.05 & 31 \\
\hline MSF & 4.48 & 0.18 & 4.11 & 4.87 & 31 \\
\hline BL & 2.77 & 0.25 & 2.3 & 3.2 & 31 \\
\hline RAP & 12.77 & 0.61 & 11.63 & 4.2 & 31 \\
\hline DL & 25.62 & 0.78 & 24.04 & 27.64 & 31 \\
\hline $\mathrm{M}^{1} \mathrm{~W}$ & 1.92 & 0.09 & 1.74 & 2.12 & 31 \\
\hline $\mathrm{M}^{\prime} \mathrm{L}$ & 2.29 & 0.12 & 2.09 & 2.59 & 31 \\
\hline $\mathrm{P}^{3} \mathrm{~W}$ & 1.83 & 0.17 & 1.17 & 2.13 & 31 \\
\hline $\mathrm{P}^{3} \mathrm{~L}$ & 2.32 & 0.14 & 1.9 & 2.52 & 31 \\
\hline $\mathrm{P}^{4} \mathrm{~W}$ & 2.16 & 0.1 & 1.96 & 2.38 & 31 \\
\hline $\mathrm{P}^{4} \mathrm{~L}$ & 2.4 & 0.13 & 1.99 & 2.66 & 31 \\
\hline CBL & 33.2 & 0.79 & 31.71 & 35.19 & 31 \\
\hline $\mathrm{M}^{1}-\mathrm{M}^{1}$ & 7.34 & 0.52 & 5.13 & 7.96 & 31 \\
\hline $\mathrm{P}^{3}-\mathrm{P}^{3}$ & 5.2 & 0.51 & 4.43 & 6.81 & 31 \\
\hline $\mathrm{P}^{4}-\mathrm{P}^{4}$ & 6.33 & 0.55 & 4.89 & 7.6 & 31 \\
\hline BS & 13.86 & 0.55 & 12.72 & 14.81 & 31 \\
\hline POZ & 14.54 & 0.44 & 13.68 & 15.42 & 31 \\
\hline $\mathrm{C}_{-} \mathrm{M}^{1}$ & 11.56 & 0.35 & 10.56 & 12.16 & 31 \\
\hline $\mathrm{C} \mathrm{B}^{2}$ & 12.67 & 0.46 & 11.29 & 13.68 & 31 \\
\hline HBL & 88.91 & 4.65 & 78.98 & 97.76 & 31 \\
\hline EAR & 17.19 & 1.07 & 4.26 & 19.24 & 31 \\
\hline FA & 72.85 & 1.99 & 67.88 & 77.32 & 31 \\
\hline TIBIA & 30.06 & 1.18 & 27.47 & 32.52 & 31 \\
\hline P2 & 34.48 & 1.6 & 30.58 & 37.31 & 31 \\
\hline P2_1 & 9.05 & 0.66 & 7.96 & 10.49 & 31 \\
\hline P3 & 50.43 & 1.47 & 47.17 & 53.33 & 31 \\
\hline P3_1 & 38.01 & 1.29 & 35.37 & 40.08 & 31 \\
\hline P4 & 48.08 & 1.79 & 44.25 & 51.92 & 31 \\
\hline P4_1 & 29.63 & 1.18 & 26.82 & 31.46 & 31 \\
\hline P5 & 50.5 & 1.96 & 46.92 & 55.22 & 31 \\
\hline P5_1 & 24.34 & 0.83 & 22.47 & 25.61 & 31 \\
\hline
\end{tabular}




\begin{tabular}{|c|c|c|c|c|c|}
\hline q Thoopterus suhaniahae sp. nov. & Mean & $\begin{array}{l}\text { Standard } \\
\text { deviation }\end{array}$ & Minimum & Maximum & $\mathrm{N}$ \\
\hline GSL & 37.1 & 0.81 & 35.82 & 38.28 & 14 \\
\hline ZB & 23.93 & 0.83 & 22.1 & 25.23 & 14 \\
\hline HB & 12.78 & 0.93 & 11.34 & 14.19 & 14 \\
\hline RL & 9.2 & 0.48 & 8.54 & 10.01 & 14 \\
\hline $\mathrm{IO}$ & 8.24 & 0.28 & 7.51 & 8.58 & 14 \\
\hline $\mathrm{C}^{1}-\mathrm{C}^{1}$ & 7.68 & 0.5 & 7.23 & 7.83 & 14 \\
\hline POW & 7.51 & 0.19 & 7.2 & 7.91 & 14 \\
\hline LOP & 18.84 & 0.57 & 18.1 & 20.03 & 14 \\
\hline MSF & 4.64 & 0.29 & 4.23 & 5.42 & 14 \\
\hline $\mathrm{BL}$ & 3 & 0.34 & 2.42 & 3.53 & 14 \\
\hline RAP & 14.11 & 0.59 & 13.12 & 14.95 & 14 \\
\hline DL & 27.67 & 0.82 & 25.58 & 28.91 & 14 \\
\hline $\mathrm{M}^{1} \mathrm{~W}$ & 2.16 & 0.11 & 1.93 & 2.37 & 14 \\
\hline $\mathrm{M}^{1} \mathrm{~L}$ & 2.52 & 0.13 & 2.23 & 2.75 & 14 \\
\hline $\mathrm{P}^{3} \mathrm{~W}$ & 1.95 & 0.24 & .9 & 2.13 & 14 \\
\hline $\mathrm{P}^{3} \mathrm{~L}$ & 2.48 & 0.2 & 1.99 & 2.84 & 14 \\
\hline $\mathrm{P}^{4} \mathrm{~W}$ & 2.37 & 0.1 & 2.22 & 2.61 & 14 \\
\hline $\mathrm{P}^{4} \mathrm{~L}$ & 2.64 & 0.11 & 2.48 & 2.87 & 14 \\
\hline CBL & 35.57 & 0.71 & 34.74 & 36.86 & 14 \\
\hline $\mathrm{M}^{1}-\mathrm{M}^{1}$ & 8.21 & 0.3 & 7.78 & 8.73 & 14 \\
\hline $\mathrm{P}^{3}-\mathrm{P}^{3}$ & 5.61 & 0.25 & 5.21 & 6.07 & 14 \\
\hline $\mathrm{P}^{4}-\mathrm{P}^{4}$ & 7.18 & 0.31 & 6.5 & 7.53 & 14 \\
\hline BS & 15.19 & 0.62 & 14.05 & 16.04 & 14 \\
\hline POZ & 5.58 & 0.38 & 14.95 & 16.03 & 14 \\
\hline C_M $M^{1}$ & 12.76 & 0.29 & 12.1 & 13.17 & 14 \\
\hline $\mathrm{C}_{-} \mathrm{M}^{2}$ & 14.01 & 0.59 & 12.52 & 14.82 & 14 \\
\hline HBL & 94.91 & 3.9 & 86.02 & 101.11 & 14 \\
\hline EAR & 17.64 & 0.89 & 16.62 & 19.5 & 14 \\
\hline FA & 76.44 & 1.64 & 73.03 & 78.47 & 14 \\
\hline TIBIA & 31.47 & 1.17 & 29.85 & 33 & 14 \\
\hline P2 & 37.22 & 1.42 & 34.5 & 39.54 & 14 \\
\hline P2_1 & 10.08 & 0.38 & 9.58 & 10.66 & 14 \\
\hline P3 & 54.24 & 1.43 & 51.07 & 56.63 & 14 \\
\hline P3_1 & 39.45 & 1.47 & 36.64 & 41.2 & 14 \\
\hline P4 & 50.35 & 1.2 & 47.39 & 51.94 & 14 \\
\hline P4_1 & 30.34 & 1 & 28.7 & 32.18 & 14 \\
\hline P5 & 52.22 & 1.79 & 48.66 & 55.17 & 14 \\
\hline P5_1 & 25.51 & 0.83 & 23.96 & 27.2 & 14 \\
\hline
\end{tabular}




\begin{tabular}{|c|c|c|c|c|c|}
\hline đT Thoopterus nigrescens & Mean & $\begin{array}{l}\text { Standard } \\
\text { deviation }\end{array}$ & Minimum & Maximum & $\mathrm{N}$ \\
\hline GSL & 35.33 & 1.2 & 32.55 & 37.67 & 32 \\
\hline ZB & 22.13 & 1.06 & 19.39 & 23.71 & 32 \\
\hline $\mathrm{HB}$ & 1.81 & 0.44 & 10.88 & 12.5 & 32 \\
\hline $\mathrm{RL}$ & 8.65 & 0.57 & 7.22 & 9.61 & 32 \\
\hline IO & 7.5 & 0.31 & 6.4 & 7.94 & 32 \\
\hline $\mathrm{C}^{1}-\mathrm{C}^{1}$ & 7.05 & 0.41 & 6.18 & 7.76 & 32 \\
\hline POW & 6.98 & 0.38 & 6.43 & 7.8 & 32 \\
\hline LOP & 17.76 & 0.64 & 16.57 & 18.76 & 32 \\
\hline MSF & 4.47 & 0.2 & 4.19 & 4.92 & 32 \\
\hline $\mathrm{BL}$ & 2.78 & 0.22 & 2.26 & 3.31 & 32 \\
\hline RAP & 3.29 & 1 & 10.43 & 15 & 32 \\
\hline $\mathrm{DL}$ & 26.33 & 1.31 & 2.78 & 28.05 & 32 \\
\hline $\mathrm{M}^{1} \mathrm{~W}$ & 1.98 & 0.11 & 1.79 & 2.15 & 32 \\
\hline $\mathrm{M}^{1} \mathrm{~L}$ & 2.28 & 0.5 & 1.84 & 2.53 & 32 \\
\hline $\mathrm{P}^{3} \mathrm{~W}$ & 1.93 & 0.23 & 1.35 & 2.53 & 32 \\
\hline $\mathrm{P}^{3} \mathrm{~L}$ & 2.34 & 0.25 & 1.41 & 2.75 & 32 \\
\hline $\mathrm{P}^{4} \mathrm{~W}$ & 2.22 & 0.7 & 1.91 & 2.62 & 32 \\
\hline $\mathrm{P}^{4} \mathrm{~L}$ & 2.47 & 0.4 & 2.27 & 2.75 & 32 \\
\hline CBL & 33.97 & 1.11 & 31.53 & 35.89 & 32 \\
\hline $\mathrm{M}^{1}-\mathrm{M}^{1}$ & 7.32 & 0.66 & 5.15 & 7.98 & 32 \\
\hline $\mathrm{P}^{3}-\mathrm{P}^{3}$ & 5.12 & 0.45 & 4.21 & 6.4 & 32 \\
\hline $\mathrm{P}^{4}-\mathrm{P}^{4}$ & 6.45 & 0.41 & 5.46 & 7.1 & 32 \\
\hline BS & 14.15 & 0.49 & 13.21 & 15.03 & 32 \\
\hline POZ & 14.75 & 0.37 & 14.08 & 15.67 & 32 \\
\hline C_M ${ }^{1}$ & 1.92 & 0.53 & 10.89 & 13.27 & 32 \\
\hline C_M ${ }^{2}$ & 13.02 & 0.54 & 11.9 & 13.79 & 32 \\
\hline HBL & 92.12 & 6.94 & 78.55 & 105.12 & 32 \\
\hline EAR & 17.74 & 0.98 & 16.17 & 19.97 & 32 \\
\hline FA & 74.25 & 3.47 & 66.65 & 79.36 & 32 \\
\hline TIBIA & 31.02 & 1.99 & 25.84 & 35.52 & 32 \\
\hline P2 & 35.73 & .83 & 32.26 & 38.26 & 32 \\
\hline P2_1 & 9.44 & 0.91 & 7.93 & 11.72 & 32 \\
\hline P3 & 51.35 & 2.16 & 47.89 & 55.49 & 32 \\
\hline P3_1 & 38.7 & .52 & 35.35 & 41.31 & 32 \\
\hline P4 & 48.94 & 1.88 & 44.24 & 52.77 & 32 \\
\hline P4_1 & 30.33 & 1.23 & 27.05 & 32.57 & 32 \\
\hline P5 & 51.75 & 1.88 & 46.02 & 55.51 & 32 \\
\hline P5_1 & 24.84 & 1.01 & 22.59 & 26.62 & 32 \\
\hline
\end{tabular}




\begin{tabular}{|c|c|c|c|c|c|}
\hline đT Thoopterus suhaniahae sp. nov. & Mean & $\begin{array}{l}\text { Standard } \\
\text { deviation }\end{array}$ & Minimum & Maximum & $\mathrm{N}$ \\
\hline GSL & 38.85 & 0.81 & 37.21 & 40.55 & 22 \\
\hline ZB & 25.12 & 0.77 & 23.76 & 26.35 & 22 \\
\hline HB & 12.77 & 0.98 & 10.41 & 4.6 & 22 \\
\hline RL & 9.54 & 0.46 & 8.95 & 10.75 & 22 \\
\hline IO & 8.71 & 0.4 & 7.89 & 9.34 & 22 \\
\hline $\mathrm{C}^{1}-\mathrm{C}^{1}$ & 8.06 & 0.29 & 7.57 & 8.58 & 22 \\
\hline POW & 7.62 & 0.31 & 7.17 & 8.26 & 22 \\
\hline LOP & 19.6 & 0.57 & 18.37 & 20.92 & 22 \\
\hline MSF & 4.59 & 0.26 & 4.16 & 5.03 & 22 \\
\hline BL & 3.03 & 0.38 & 2.2 & 3.68 & 22 \\
\hline RAP & 15.32 & 0.93 & 13.63 & 17.01 & 22 \\
\hline DL & 29.33 & 0.77 & 28.03 & 31.06 & 22 \\
\hline $\mathrm{M}^{1} \mathrm{~W}$ & 2.24 & 0.07 & 2.08 & 2.33 & 22 \\
\hline $\mathrm{M}^{1} \mathrm{~L}$ & 2.57 & 0.17 & 2.3 & 2.92 & 22 \\
\hline $\mathrm{P}^{3} \mathrm{~W}$ & 2.15 & 0.16 & 1.96 & 2.55 & 22 \\
\hline $\mathrm{P}^{3} \mathrm{~L}$ & 2.65 & 0.2 & 2.02 & 2.85 & 22 \\
\hline $\mathrm{P}^{4} \mathrm{~W}$ & 2.48 & 0.18 & 2.19 & 2.9 & 22 \\
\hline $\mathrm{P}^{4} \mathrm{~L}$ & 2.68 & 0.1 & 2.44 & 2.8 & 22 \\
\hline $\mathrm{CBL}$ & 37.25 & 0.88 & 35.51 & 39.28 & 22 \\
\hline $\mathrm{M}^{1}-\mathrm{M}^{1}$ & 8.37 & 0.34 & 7.68 & 8.85 & 22 \\
\hline $\mathrm{P}^{3}-\mathrm{P}^{3}$ & 5.77 & 0.37 & 5.13 & 6.36 & 22 \\
\hline $\mathrm{P}^{4}-\mathrm{P}^{4}$ & 7.34 & 0.4 & 6.61 & 7.85 & 22 \\
\hline BS & 16.04 & 0.59 & 15.18 & 17.24 & 22 \\
\hline POZ & 16.19 & 0.51 & 15.6 & 17.77 & 22 \\
\hline C_. ${ }^{1}$ & 13.27 & 0.29 & 12.71 & 13.73 & 22 \\
\hline $\mathrm{C}_{-} \mathrm{M}^{2}$ & 14.88 & 0.27 & 14.26 & 15.3 & 22 \\
\hline HBL & 98.47 & 4.05 & 89.35 & 109.67 & 22 \\
\hline EAR & 17.79 & 0.69 & 16.62 & 19.06 & 22 \\
\hline FA & 78.36 & 1.87 & 74.42 & 81.77 & 22 \\
\hline TIBIA & 32.26 & 1.03 & 30.49 & 34.11 & 22 \\
\hline P2 & 38.03 & 0.95 & 36.13 & 39.87 & 22 \\
\hline P2_1 & 10.45 & 0.65 & 9.5 & 11.57 & 22 \\
\hline P3 & 55.23 & 1.74 & 52.37 & 57.73 & 22 \\
\hline P3_1 & 41.19 & 1.16 & 37.85 & 42.83 & 22 \\
\hline P4 & 51.97 & 1.62 & 48.72 & 54.43 & 22 \\
\hline P4_1 & 31.98 & 0.97 & 30.46 & 33.59 & 22 \\
\hline P5 & 53.51 & 1.53 & 50.27 & 55.65 & 22 \\
\hline P5_1 & 26.27 & 0.91 & 24.73 & 27.52 & 22 \\
\hline
\end{tabular}


TABLE 2 Measurements $(\mathrm{mm})$ for skull includes dentary, dental and external characters (see Material and Methods section for explanation of character codes) of adult Thoopterus spp.

Sex

Species

Sex vs. species

\begin{tabular}{|c|c|c|c|c|c|c|}
\hline Dependent Variable & $\mathrm{F}$ & $P$ & $\mathrm{~F}$ & $P$ & $\mathrm{~F}$ & $P$ \\
\hline GSL & 43.11 & 0.001 & 205.49 & 0.001 & 3.04 & 0.08 \\
\hline ZB & 14.97 & 0.001 & 163.72 & 0.001 & 2.93 & 0.09 \\
\hline HB & 0.18 & 0.67 & 53.48 & 0.001 & 0.56 & 0.46 \\
\hline RL & 8.86 & 0.001 & 56.08 & 0.001 & 0.53 & 0.47 \\
\hline IO & 18.98 & 0.001 & 208.67 & 0.001 & 6.51 & 0.01 \\
\hline $\mathrm{Cl}-\mathrm{Cl}$ & 21.99 & 0.001 & 181.59 & 0.001 & 0.42 & 0.52 \\
\hline POW & 3.74 & 0.06 & 81.26 & 0.001 & 0.03 & 0.86 \\
\hline LOP & 27.33 & 0.001 & 167.29 & 0.001 & 1.01 & 0.32 \\
\hline MSF & 0.15 & 0.7 & 5.98 & 0.02 & 0.27 & 0.61 \\
\hline $\mathrm{BL}$ & 0.01 & 0.94 & 14.87 & 0.001 & 0.01 & 0.95 \\
\hline RAP & 0.35 & 0.55 & 12.95 & 0.001 & 1.42 & 0.24 \\
\hline $\mathrm{DL}$ & 28.22 & 0.001 & 115.03 & 0.001 & 5.41 & 0.02 \\
\hline M1W & 10.03 & 0.001 & 120.9 & 0.001 & 0.4 & 0.53 \\
\hline M1L & 0.51 & 0.48 & 60.17 & 0.001 & 0.7 & 0.41 \\
\hline $\mathrm{P} 3 \mathrm{~W}$ & 10.2 & 0.001 & 12.53 & 0.001 & 1.36 & 0.25 \\
\hline P3L & 4.42 & 0.04 & 23.79 & 0.001 & 2.58 & 0.11 \\
\hline P4W & 5.25 & 0.02 & 47.04 & 0.001 & 0.18 & 0.67 \\
\hline P4L & 4.95 & 0.03 & 59.66 & 0.001 & 0.31 & 0.58 \\
\hline CBL & 35.47 & 0.001 & 182.37 & 0.001 & 3.96 & 0.05 \\
\hline M1-M1 & 0.48 & 0.49 & 60.7 & 0.001 & 0.51 & 0.48 \\
\hline P3-P3 & 0.78 & 0.38 & 31.23 & 0.001 & 0.81 & 0.37 \\
\hline P4-P4 & 2.35 & 0.13 & 68.92 & 0.001 & 0.08 & 0.78 \\
\hline MB & 20.2 & 0.001 & 153.91 & 0.001 & 6.11 & 0.02 \\
\hline BW & 19.8 & 0.001 & 157.82 & 0.001 & 5.51 & 0.02 \\
\hline C_M1 & 23.97 & 0.001 & 178.64 & 0.001 & 0.98 & 0.33 \\
\hline $\mathrm{C} 1-\mathrm{M} 2$ & 33.59 & 0.001 & 201.61 & 0.001 & 6.66 & 0.01 \\
\hline $\mathrm{TL}$ & 2.27 & 0.135 & 10.9 & 0.001 & 0.27 & 0.6 \\
\hline EAR & 1.57 & 0.213 & 1.967 & 0.164 & 0.34 & 0.56 \\
\hline FA & 9.77 & 0.002 & 47.91 & 0.001 & 0.29 & 0.59 \\
\hline TIBIA & 8.37 & 0.005 & 16.1 & 0.001 & 0.16 & 0.69 \\
\hline P2 & 9.2 & 0.003 & 56.19 & 0.001 & 0.36 & 0.55 \\
\hline P2_1 & 4.72 & 0.033 & 36.17 & 0.001 & 0.01 & 0.94 \\
\hline P3 & 4.7 & 0.033 & 70.19 & 0.001 & 0.01 & 0.98 \\
\hline P3_1 & 5.51 & 0.021 & 15.37 & 0.001 & 1.543 & 0.22 \\
\hline P4 & 11.7 & 0.001 & 47.16 & 0.001 & 0.73 & 0.4 \\
\hline P4_1 & 7.45 & 0.008 & 23.17 & 0.001 & 1.48 & 0.23 \\
\hline P5 & 7.49 & 0.008 & 17.57 & 0.001 & 0.05 & 0.82 \\
\hline P5_1 & 10.5 & 0.002 & 40.74 & 0.001 & 0.32 & 0.57 \\
\hline
\end{tabular}


(30.49-34.11) Vs 31.47 (29.55-33.00); P2 38.03 (36.13$39.87)$ v. 37.22 (34.50-38.54); P2_1 10.45 (9.50-11.57) v. 10.08 (9.58-10.66) (Table 1).

External measurements of $T$. suhaniahae sp. nov. are larger than $T$. nigrescens as follows: the female head and body length 94.91 (86.02-101.11) v. 89.69 (78.98-107.66), ear length $17.63(16.62-19.50)$ v. 17.19 (14.26-19.24), forearm length $76.43(73.03-78.47) \mathrm{v}$. 72.84 (67.88-77.32); the male head and body length $98.47(89.35-109.67)$ v. 92.12 (78.55-105.12), ear length 17.79 (16.62-19.06) v. 17.74 (16.17-19.97), FA 78.36 (74.42-81.77) v. 74.25 (66.65-79.36).

Mean, standard deviation, minimum, maximum values and samples size for each species and different sexes are presented in Table 1 for cranial, dental, mandibular and external characters. MANOVA results (see Table 2 for details) indicate that nearly all characters differed significantly between the sexes. Characters that not were significantly influenced by sex $(P>0.05)$ were: braincase height (HB), bulla length (BL), ramus angular process (RAP), upper first molar length $\left(\mathrm{M}^{1} \mathrm{~L}\right)$, distance between upper first molars $\left(\mathrm{M}^{1}-\mathrm{M}^{1}\right)$, upper third premolars $\left(\mathrm{P}^{3}-\mathrm{P}^{3}\right)$, and upper fourth premolar $\left(\mathrm{P}^{4}-\mathrm{P}^{4}\right)$, ear length $(\mathrm{EAR})$ and head and body length (HBL) (Table 2). All characters showed significant differences between $T$. nigrescens and $T$. suhaniahae sp. nov. at $P<0.001$; exceptions are MSF and ear length.

For most characters there were no significant interactions between sex and species (Table 2). Weak interactions between sex and species were found for interorbital width (IO), lower canine to second molar distance $\left(\mathrm{C}-\mathrm{M}_{2}\right)(P<0.01)$ and for condylobasal length $(\mathrm{CBL})$, dentary length (DL), braincase width (BW) and mastoid breadth $(\mathrm{MB})(P<0.05)$.

\section{MULTIVARIATE ANALYSES}

DFA for 26 skull dental and dentary characters and 12 external characters were run separately. The DFA was run using a reduced set of five selected characters to reduce the effect of over fitting data. Five variables were selected to minimize Wilk's lambda. These subsets provided similar cluster values in discriminant function space as had been observed in the full set of 26 skull, dental and dentary characters. The DFA for males and females extracted a highly significant function that did not misclassify any individuals of $T$. nigrescens and T. suhaniahae sp. nov.

The DFA of male specimens contrasted $T$. suhaniahae sp. nov. from Sulawesi, Wowoni and Talaud Islands with $T$. nigrescens collected from Sulawesi, Sula, Wowoni and Buton Island. The five skull and external character for male selected to minimize Wilk's lambda (Kitchener et.al. 1995) are $\mathrm{M}^{1} \mathrm{~W}, \mathrm{IO}, \mathrm{POW}, \mathrm{POZ}, \mathrm{M}^{1}-\mathrm{M}^{1}$ and P3, P2_1, P5, P3_1, HBL. The analyses of female specimens contrasted T. suhaniahae sp. nov. from Sulawesi and second, T. nigrescens collected from Sulawesi, Buton, Sula,
Wowoni Islands. The five skull and external characters selected to minimize Wilk's lambda female are $\mathrm{C}^{1}-\mathrm{C}^{1}$, GSL, HB, MSF, Mㄴ and P3, P5, P2_1, FA, HBL.

The separate DFA for males and females extracted the highly significant function (Function 1) and classified $100 \%$ individuals correctly to species. Variables loading most heavy $(>0.5)$ on these functions were POW, P3, P5, P3_1, P2_1 (males) and $\mathrm{C}^{1}-\mathrm{C}^{1}, \mathrm{P} 3, \mathrm{P} 5, \mathrm{HBL}$ (females).

\section{SYSTEMATICS}

\section{Family Pteropodidae}

\section{Genus Thoopterus Matschie, 1899}

\section{TYPE SPECIES}

Cynopterus marginatus var. nigrescens Gray, 1870.

\section{DIAGNOSIS}

Postorbital foramina absent; incisors complete, upper canine grooved, cheek teeth $4 / 5, \mathrm{P}_{4}$ and $\mathrm{M}_{1}$ broad, quadrate with large surface and grooved upper canines without cingulum cusps; tail reduced to a rudiment, membranes from second toe with longer fur.

\section{Thoopterus nigrescens (Gray, 1870)}

Figures 2, 3

\section{MATERIAL EXAMINED}

\section{Holotype}

Indonesia: North Moluccas: $q$ skin and skull, Morotai, A.R. Wallace (BMNH 62.10.21.6).

\section{DIAGNOSIS}

No postorbital foramina, incisors $2 / 2-2 / 2$, upper canine grooved, check teeth $4 / 5, \mathrm{P}_{4}$ and $\mathrm{M}_{2}$ extremely broad, quadrate, with large surface cusps, and the inner ridge quite obsolete. Tail rudimentary, membranes from second toe' (Andersen 1912).

\section{DESCRIPTION}

Description follows Andersen (1912): fur much longer than in Cynopterus, back warm prout's brown, under fur pale drab grey, head similar but slightly darker, nape neck paler, under parts from chin to interfemoral, hair brown, slightly shaded with a warmer brown on flanks and sides of breast and belly, the metacarpal and phalanges dark brown. Rostrum unmodified; premaxillae slender, tapering above in simple contact interiorly; row of upper incisors in sharp profile of rostrum (one canine covering the other) partly hidden between canines. Front of orbits above back of $\mathrm{P}^{4}$, maxillary tooth row not extending beyond ventral margin of orbit. Sagittal crest fully developed, but low.

\section{DISTRIBUTION}

Sulawesi, Talaud, Sula, Buton, Wowoni, Morotai 


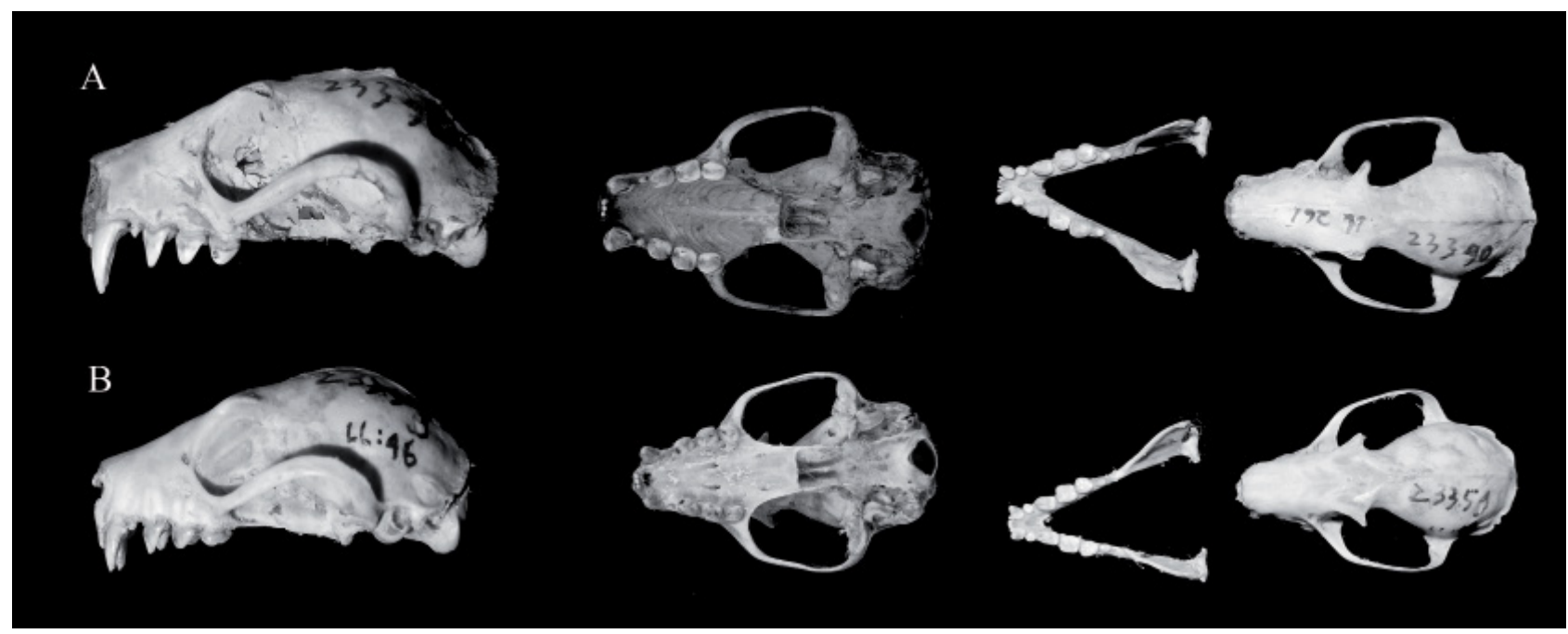

FIGURE 2 Photograph of the skulls of Thoopterus suhaniahae (a) and T. nigrescens (b), showing the lower mandible and cranium in dorsal, ventral and lateral views.

Island.

\section{Thoopterus suhaniahae sp. nov.}

Figures 2, 3, 6

\section{MATERIAL EXAMINED}

\section{Holotype}

Indonesia: Central Sulawesi: MZB 23390: , LL 261, Lore Lindu National Park, Watumeta $\left(1^{\circ} 23^{\prime} 57^{\prime \prime S}\right.$, $\left.120^{\circ} 18^{\prime} 07^{\prime \prime E}, 1125 \mathrm{~m}\right)$, collected on 26 July 2000 by I. Maryanto and M. Yani.

\section{Paratypes}

Indonesia: 12 : $\mathrm{OZB}$ 23360, MZB 23363, MZB 23365, MZB 23369, MZB 23372, MZB 23374, MZB 23383, MZB 23384, MZB 23386, MZB 23387, MZB 23376, MZB 23478 (see Appendix 1); 24 స̊: MZB 13378, MZB 15326, MZB 15329, MZB 17812, MZB 23361, MZB 23364, MZB 23367, MZB 23368, MZB 23370, MZB 23371, MZB 23373, MZB 23377, MZB 23378, MZB 23379, MZB 23380, MZB 23381, MZB 23382, MZB 23385, MZB 23389, MZB 23391, MZB 23392, MZB 23393, MZB 27725, MZB 27726 (localities details see Appendix 1).

\section{DIAGNOSIS}

Thoopterus suhaniahae sp. nov. is diagnosed by cranial, dental, and external characters and by combinations of these (see discriminant function analysis and univariate plots, Figures 4, 5). Compared to $T$. nigrescens, metacarpal 3 of $T$. suhaniahae sp. nov. is longer relative to metacarpal 4 phalanx 1 ; $\mathrm{ZB}$ is wider relative to skull length in $T$. suhaniahae sp. nov. and palatal length is longer relative to postorbital width than T. nigrescens (Figure 5).

\section{DESCRIPTION}

Skull, dentary, dentition. Compared with $T$. nigrescens cranium of $T$. suhaniahae sp. nov., is broader and has a shorter rostrum. There are no postorbital foramina in either taxon. The mandible is more robust underneath the mandibular tooth row. In T. suhaniahae sp. nov. the second upper incisors are slightly shorter than first upper incisors. There is a broad and deep vertical groove on the antero-medial surface of the upper canine. The canine and second incisors are separated by auricle diastema. $\mathrm{P}_{1}$ is more massive than $\mathrm{P}^{1} ; \mathrm{M}_{2}$ nearly the same size as $\mathrm{P}_{1} ; \mathrm{M}^{1}$ smaller than $\mathrm{M}_{1} ; \mathrm{P}_{4}$ heavier than $\mathrm{M}_{1}$; and $\mathrm{P}^{4}$ smaller than $\mathrm{P}_{4}$.

External characters. Tail absent or reduced to a rudiment but usually more conspicuous than in $T$. nigrescens. Other external characters are as described for T. nigrescens by Andersen (1912), except for the interfemoral membranes which differ in having a quarter to half of the under face of the uropatagium sparsely furred and the calcar wider in T. suhaniahae sp. nov. is $10-11 \mathrm{~mm}$ (measured from tibia) v. $7-8 \mathrm{~mm}$ in T. nigrescens.

Pelage. The dorsum of $T$. suhaniahae sp. nov. is brown, under fur grayish brown, and head, forehead, forearm and rump darker brown. The under parts from chest to abdomen are brownish gray, from chin to interfemoral brown, and the flanks and sides of breast and belly are slightly shaded with a warmer brown. The ear and membranes are unmarked; the metacarpals and phalanges are dark brown. The dorsal fur of both T. suhaniahae sp. nov. and T. nigrescens is $10-13 \mathrm{~mm}$ long.

Glans penis. Seen under a scanning electron microscope, the urethal aperture of the glans penis of T. suhaniahae sp. nov. lacks the conspicuous scale-like projection seen in T. nigrescens (Figure 3). 


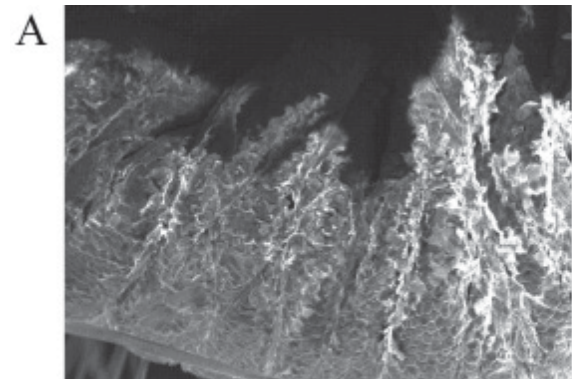

Type JSM-5000; magnification $\mathrm{x}$ $150 \mathrm{ACCV} 10 \mathrm{kV}$; width 880 um

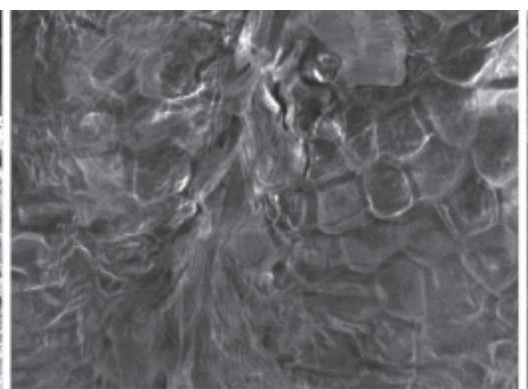

Type JSM-5000; magnification x 750 ACCV $10 \mathrm{kV}$ : width $176 \mathrm{um}$

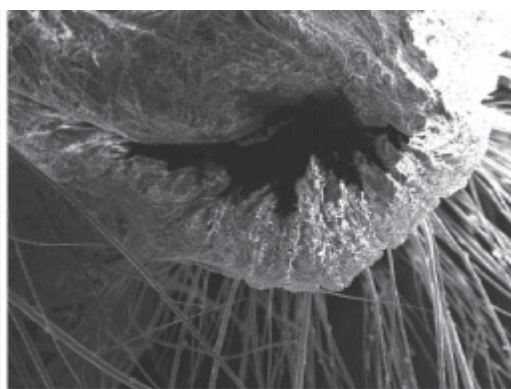

Type JSM-5000; magnification $\mathrm{x}$ $50 \mathrm{ACCV} 10 \mathrm{kV}$; width $2.64 \mathrm{~mm}$

B

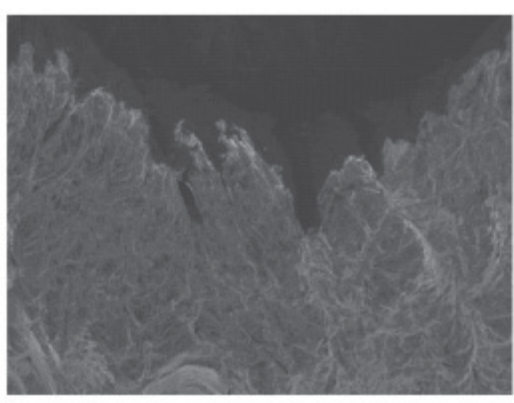

Type JSM-5000; magnification x 150; ACCV $10 \mathrm{kV}$ width $880 \mathrm{um}$

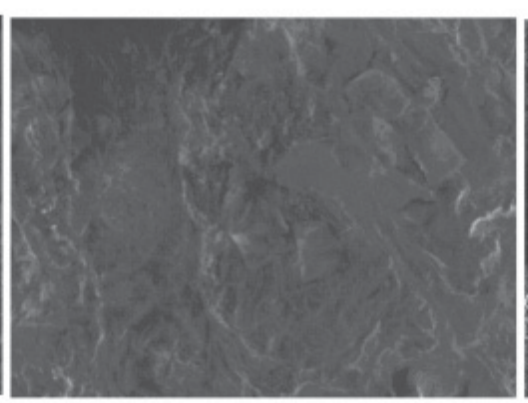

Type JSM-5000; magnification x 750; ACCV $10 \mathrm{kV}$ width $176 \mathrm{um}$

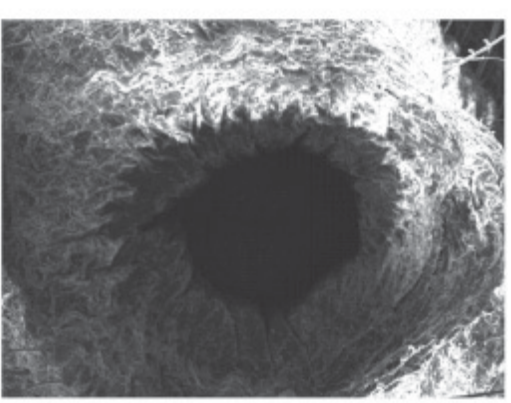

Type JSM-5000; magnification x 35; ACCV $10 \mathrm{kV}$ width $3.77 \mathrm{~mm}$

FIGURE 3 Scanning electron microscope photograph of glans penis morphology of Thoopterus nigrescens (a) and T. suhaniahae sp. nov. (b).

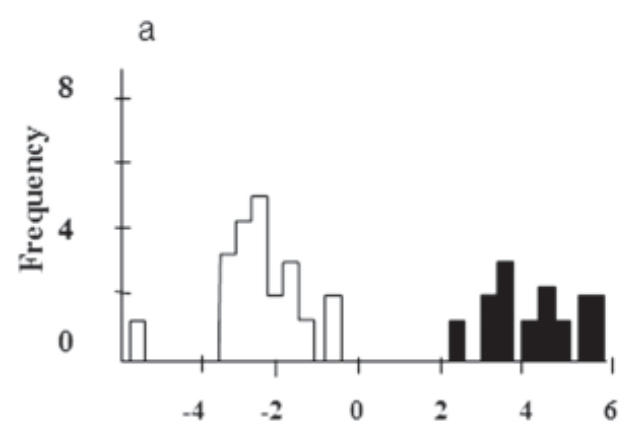

C

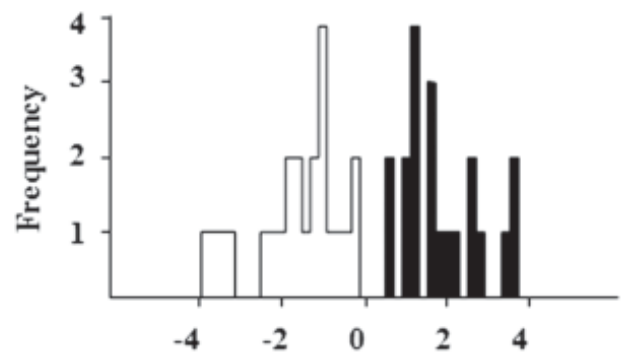

b

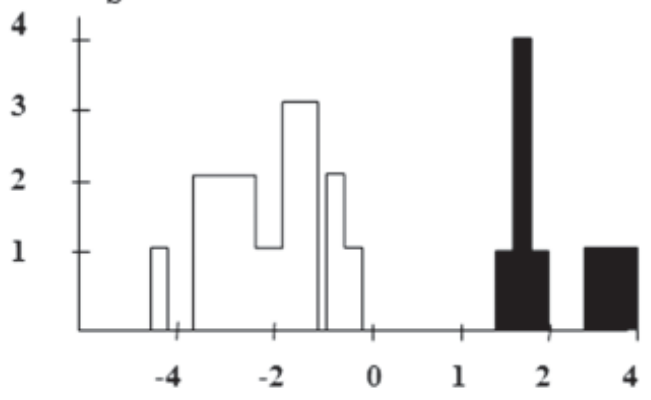

d

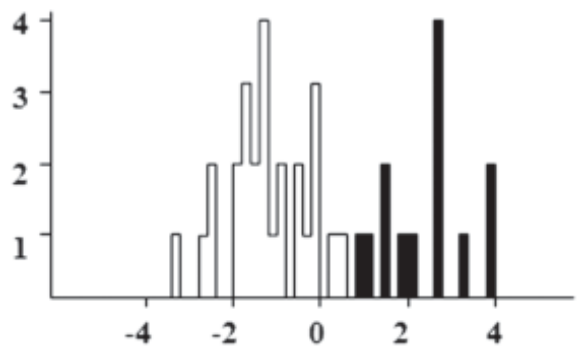

\section{Function 1}

FIGURE 4 Dendrograms of discriminant function values for male and female between Thoopterus nigrescens (white) and T. suhaniahae sp. nov. (black) (a), male skull characters; (b), female skull characters; (c), male external characters; and (d) female external characters. 

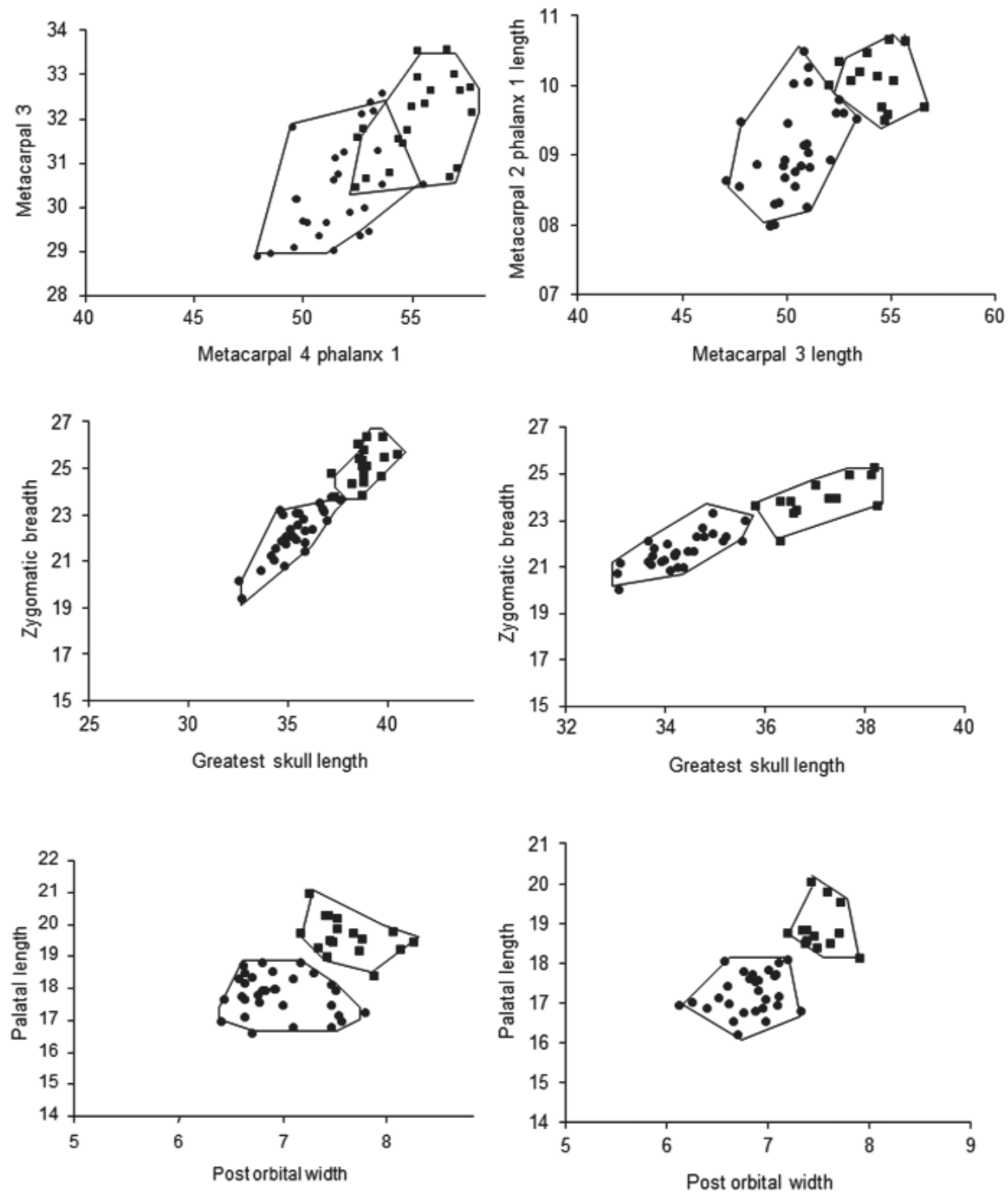

FIGURE 5 Univariate plots of palatal length and postorbital width, and greatest skull length and zygomatic breadth, metacarpal 3 and metacarpal 4 phalanx 1 (in $\mathrm{mm}$ ) of male and female Thoopterus nigrescens $(\bullet$ ) and T. suhaniahae sp. nov. (匹).

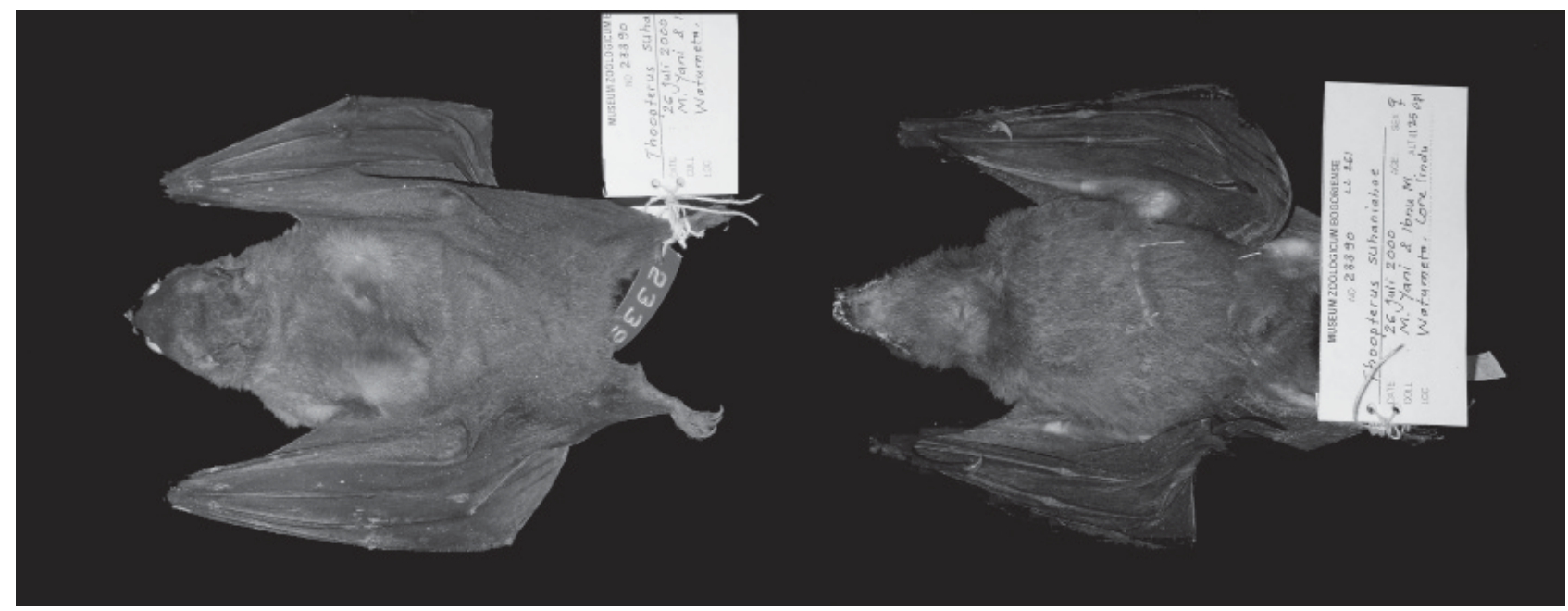

FIGURE 6 Dorsal and ventral views of the holotype (MZB 23390) of Thoopterus suhaniahae sp. nov. 
TABLE 3 Standardised and unstandardised values of the canonical variate function for the two species group of the genera of Thoopterus. a) cranium, dentary and b) external characters.

a) Skull, dental, and dentary characters

Function 1

\begin{tabular}{lll|lll} 
C1 & Standardised & Unstandardised & \multicolumn{1}{l}{ S } & Standardised & Unstandardised \\
C1-M2 & 0.44 & 0.9508127 & C1-C1 & 0.58 & 2.84 \\
IO & 0.41 & 1.2561244 & GSL & 0.465 & 0.64 \\
POW & 0.55 & 1.526736 & HB & 0.34 & 0.61 \\
POZ & 0.46 & 1.0838556 & POW & 0.29 & 1.14 \\
P4W & 0.34 & 1.9432024 & M1L & 0.3 & 2.4 \\
(Constant) & & -55.07 & & & -63.42 \\
\hline
\end{tabular}

b) External characters

Function 1

\begin{tabular}{lll|} 
P3 & Standardised & Unstandardised \\
P2_1 & 0.955 & 0.567 \\
P5 & 0.521 & 0.638 \\
P3_1 & -0.829 & -0.468 \\
HBL & 0.553 & 0.421 \\
(Constant) & 0.231 & 0.393 \\
\hline
\end{tabular}

Function 1

$\begin{array}{ll}\text { Standardised } & \text { Unstandardised } \\ 0.819 & 0.584 \\ -1.023 & -0.584 \\ 0.452 & 0.973 \\ 0.452 & 0.261 \\ 0.568 & 0.126 \\ & -38.22\end{array}$

\section{HABITAT}

Thoopterus suhaniahae sp. nov. is common in Central Sulawesi at elevations of $600-1,000 \mathrm{~m}$. Specimens were also captured at $1,930 \mathrm{~m}$ at Rorekatimbu, Lore Lindu National Park, Central Sulawesi, and at $60 \mathrm{~m}$ asl at Wowoni Island. Thoopterus suhaniahae sp. nov. has been collected sympatrically with $T$. nigrescens, Rousettus celebensis, Cynopterus luzoniensis, Cynopterus minutus (a new record for Sulawesi), Chironax melanocephalus and Macroglossus minimus. The species was collected from Maros, South Sulawesi; Bakiriang-Batu, South-West Sulawesi; Dako MountainToli-Toli, Central Sulawesi; Talaud Island, North Sulawesi, and Wowoni Island, South-East Sulawesi and South-West Sulawesi.

\section{DISTRIBUTION}

Known from Sulawesi, Talaud and Wowoni Islands, Indonesia.

\section{ETYMOLOGY}

For Suhaniah (wife of Mohamad Yani), who passed away on 31 March 2000 while her husband studied in Lore Lindu National Park.

\section{REMARKS}

The new species of Thoopterus is a medium-sized fruit bat in Sulawesi and small adjacent island groups. From our observations, it is one of the most abundant bats which widely distributed on Sulawesi from 602,100 m asl.

Geographically sympatric to T. suhaniahae sp. nov., T. nigrescens is found in primary, secondary, cacao and coffee plantation forest, whereas $T$. suhaniahae sp. nov. is more common in primary forest at middle and low land altitudes.

The discovery of a second species of Thoopterus endemic to Sulawesi and adjacent islands provides further evidence that Sulawesi is a 'hot spot' of pteropodid evolution (Maryanto and Yani 2003). The majority of distribution of the new species such as Rousettus linduensis and $R$. bidens were known from Central Sulawesi which appears to emphasize once more the complex geological history of the island as a composition of parts with different origins (Maryanto and Yani 2003).

Unfortunately, ongoing large scale hunting and trapping of fruit bats in the north and parts of Central Sulawesi and elsewhere, seriously endangers the survival of this species as well as other fruit bats on these islands. 


\section{ACKNOWLEDGMENTS}

We were indebted to Duncan Neville, The Nature Conservancy Program Manager at Palu, and Darrell Kitchener, TNC Director of Conservation, for their organization of the mammal survey on Lore Lindu National Park; we also thank Edward Polard, Program Manager for conservation at TNC Palu branch. We gratefully acknowledge the support of Ir. Banjar Yulianto Laban MSc, the Director of Lore Lindu National Park, who provided us with great assistance in the field.

Thanks are extended to our field assistants Mohamad Annas, Hariyanto and Thius Jacson, who assisted in developing the trapping procedures. Expedition costs were met by a grant to The Nature Conservancy Indonesia program from the USAID /NRM2 (Nature for Resource Management 2) program, Jakarta, Indonesia.

Finally, we are indebted to D.J. Kitchener, Chris Watts, Kristofer Helgen (Smithsonian Museum), R.A. How (Western Australian Museum) for their constructive comments on an earlier version of this paper, Endang Purwaningsih from Museum Zoologicum Bogoriense for scanning electron microscopy, Ir. Koestoto Subekti, Boeadi and A. Saim for study of their specimens from Wowoni and Buton Islands.

\section{REFERENCES}

Andersen, K. (1912). Catalogue of the Chiroptera in the collection of the British Museum. Vol 1. Megachiroptera. $2^{\text {nd }}$ edition (pp 1-854). British Museum (Natural History): London. i-ci.

Bergmans, W. and Rozendaal, F.G. (1988). Notes on collections of fruit bats from Sulawesi and some off-lying islands (Mammalia, Megachiroptera). Zoologische Verhandelingen 248: $1-74$.
Gray, J.E. (1870). Catalogue of monkeys, lemurs, and fruit eating bats in the collection of the British Museum. British Museum (Natural History): London.

Hill, J.E. (1983). Bats (Mammalia: Chiroptera) from IndoAustralia. Bulletin of the British Museum Natural History (Zoology) 45: 103-208.

Kitchener, D.J., Schmitt, L.H., Strano, P., Wheeler, A, and Suyanto. A. (1995). Taxonomy of Rhinolophus simplex Andersen, 1905 (Chiroptera: Rhinolophidae) in Nusa Tenggara and Maluku, Indonesia. Records of Western Australian Museum 17: 1-28.

Kitchener, D.J, and Maharadatunkamsi (1991). Description of new species of Cynopterus (Chiroptera: Pteropodidae) from Nusa Tenggara, Indonesia. Records of Western Australian Museum 15: 307-367.

Kitchener, D.J., Packer W.C. and Maryanto. I. (1995). Systematic review of Nyctimene cephalotes and $N$. albiventer (Chiroptera: Pteropodidae) in the Maluku and Sulawesi regions, Indonesia. Records of Western Australian Museum 17: 125-142.

Kornerup, A. and Wanscher, J.H. (1984). Methuen handbook of colour. 3rd edition Methuen: London.

Maryanto, I. and Yani, M. (2001). Small mammals survey from Lore Lindu National Park, Central Sulawesi, Indonesia. TNC Country Report: Jakarta.

Maryanto, I. and Yani, M. (2003). A new species of Rousettus (Chiroptera: Pteropodidae) from Lore Lindu, Central Sulawesi. Mammal Study 28: 111-120.

Matschie, P. (1899). Die Fledermause des Berliner Museum fur Naturkunde I. Die Megachiroptera des Berliner Museum fur Naturkunde. Riemer: Berlin.

Suyanto, A., Yoneda, M., Maryanto, I., Maharadatunkamsi and Sugardjito, J. (1998). Checklist of the mammals of Indonesia: scientific name and distribution area table in Indonesia including CITES, IUCN and Indonesian category for conservation. LIPI-JICA: Bogor.

MANUSCRIPT RECEIVED 1 APRIL 2009; ACCEPTED 21 FEBRUARY 2012. 


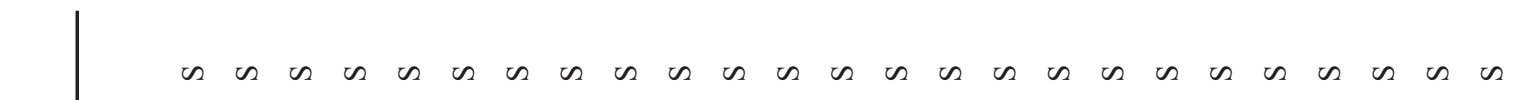

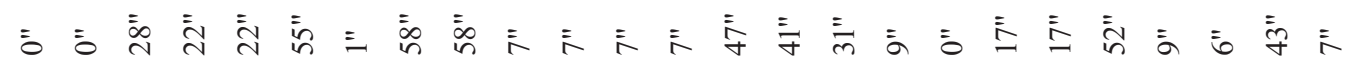

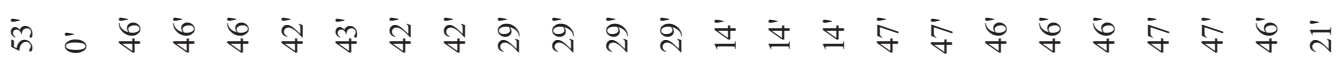

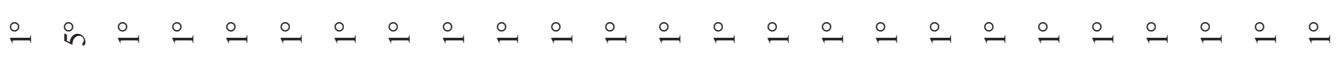

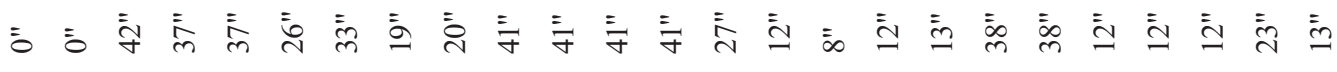

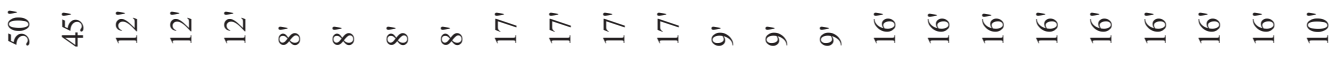

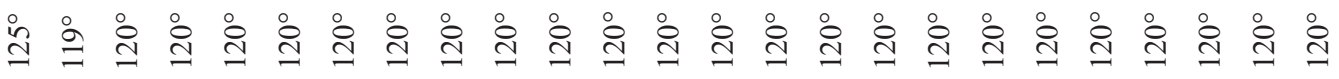

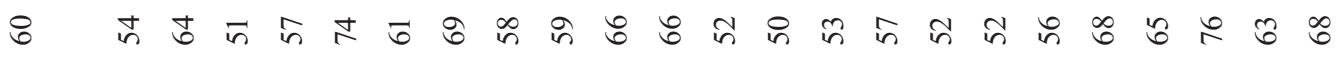
\&̊

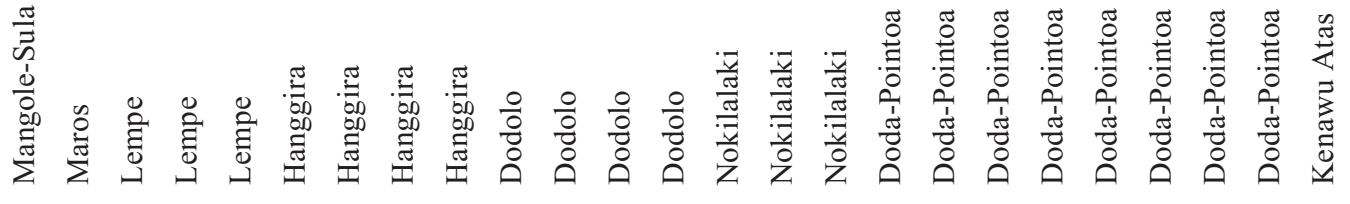
ప

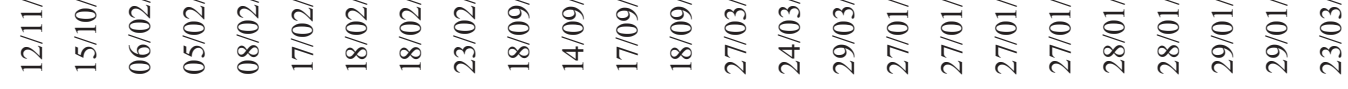

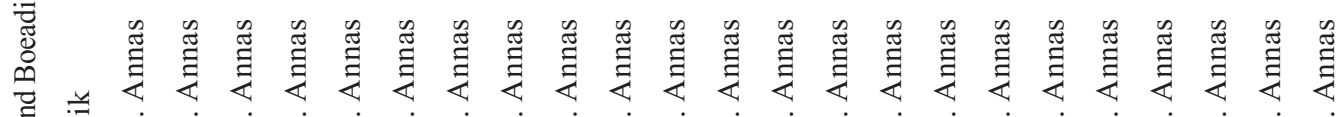

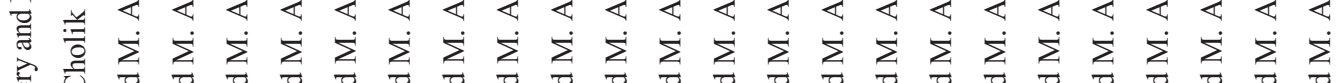

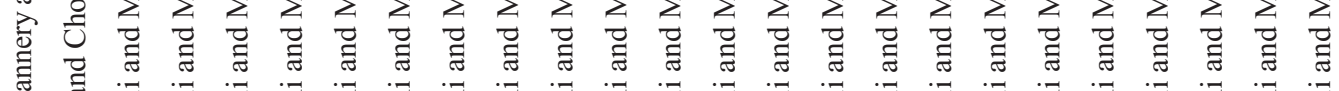

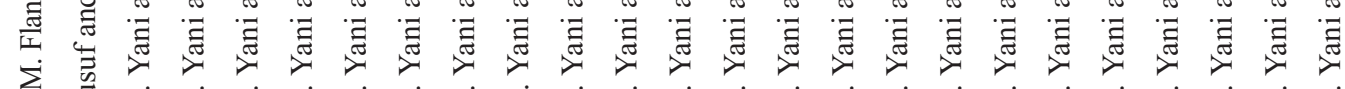
$\underset{H}{\sum} \dot{\Sigma} \dot{\Sigma} \dot{\Sigma} \dot{\Sigma} \dot{\Sigma} \dot{\Sigma} \dot{\Sigma} \dot{\Sigma} \dot{\Sigma} \dot{\Sigma} \dot{\Sigma} \dot{\Sigma} \dot{\Sigma} \dot{\Sigma} \dot{\Sigma} \dot{\Sigma} \dot{\Sigma} \dot{\Sigma} \dot{\Sigma} \dot{\Sigma} \dot{\Sigma} \dot{\Sigma} \dot{\Sigma}$

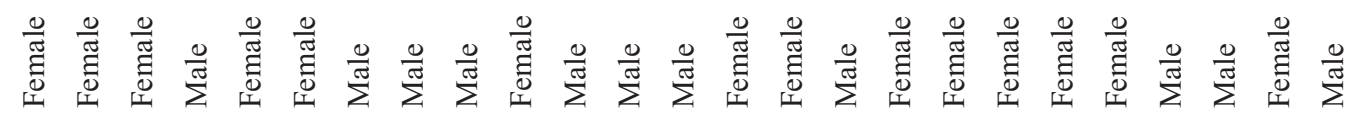

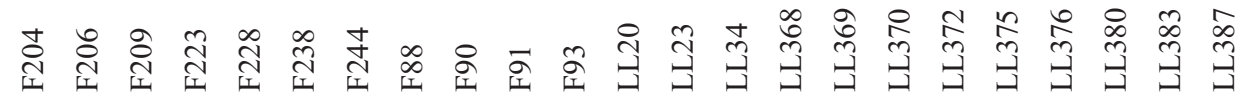




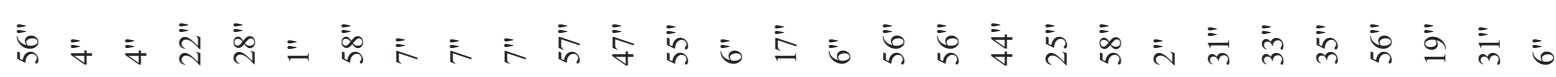

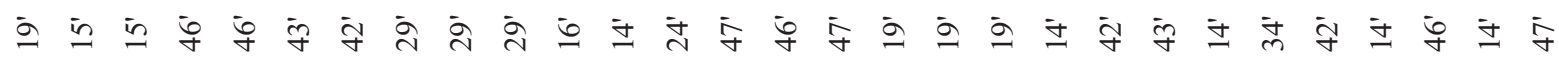

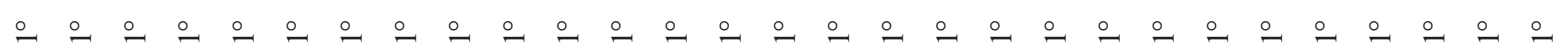

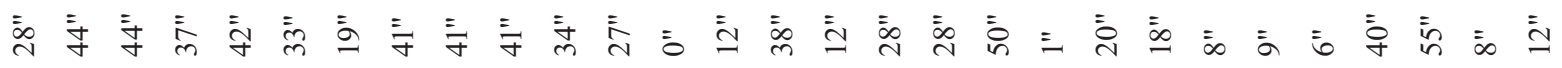

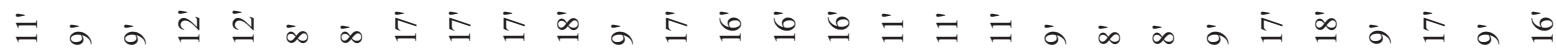

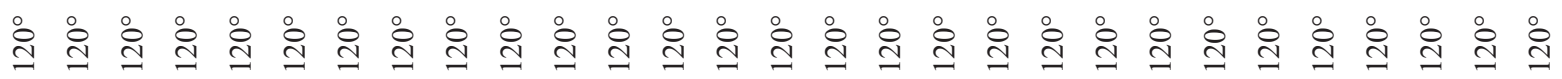

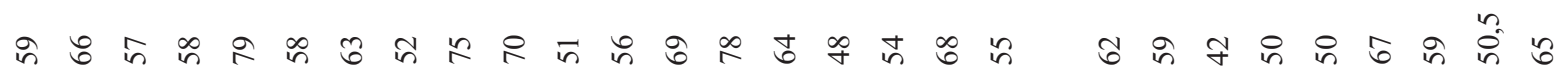
옫 च \&

๙

$\stackrel{\square}{\square}$

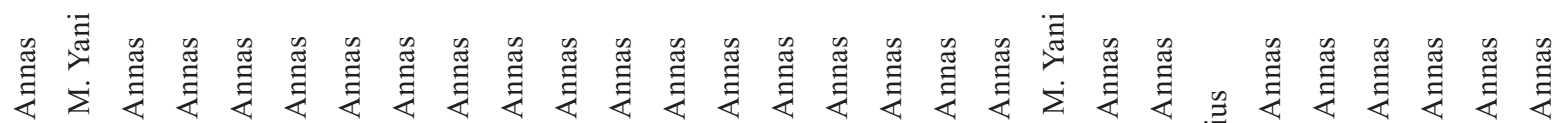

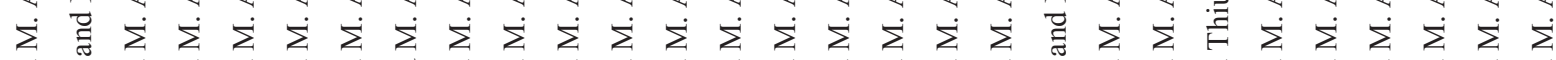

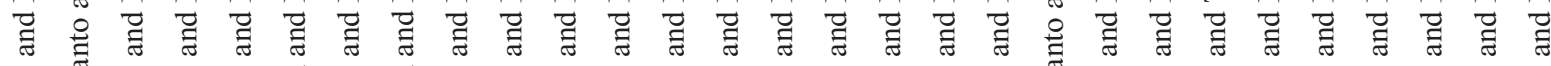

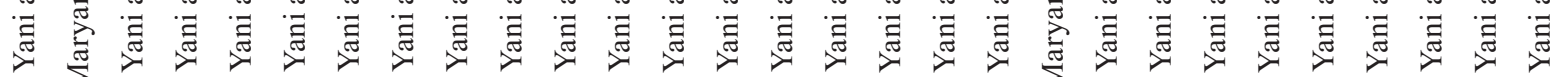
$\dot{\Sigma} \dot{\Sigma} \dot{\Sigma} \dot{\Sigma} \dot{\Sigma} \dot{\Sigma} \dot{\Sigma} \dot{\Sigma} \dot{\Sigma} \dot{\Sigma} \dot{\Sigma} \dot{\Sigma} \dot{\Sigma} \dot{\Sigma} \dot{\Sigma} \dot{\Sigma} \dot{\Sigma} \dot{\Sigma} \dot{\Sigma}$

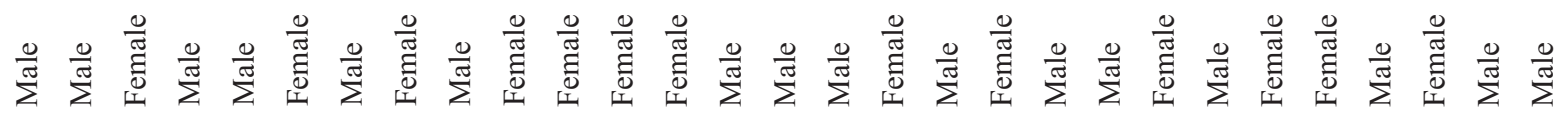

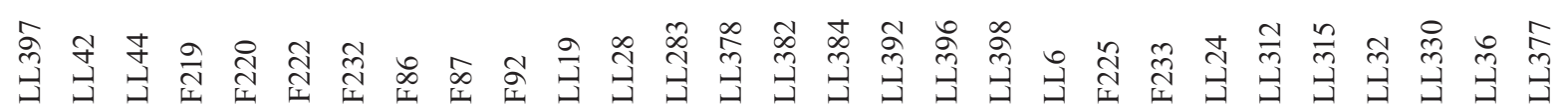




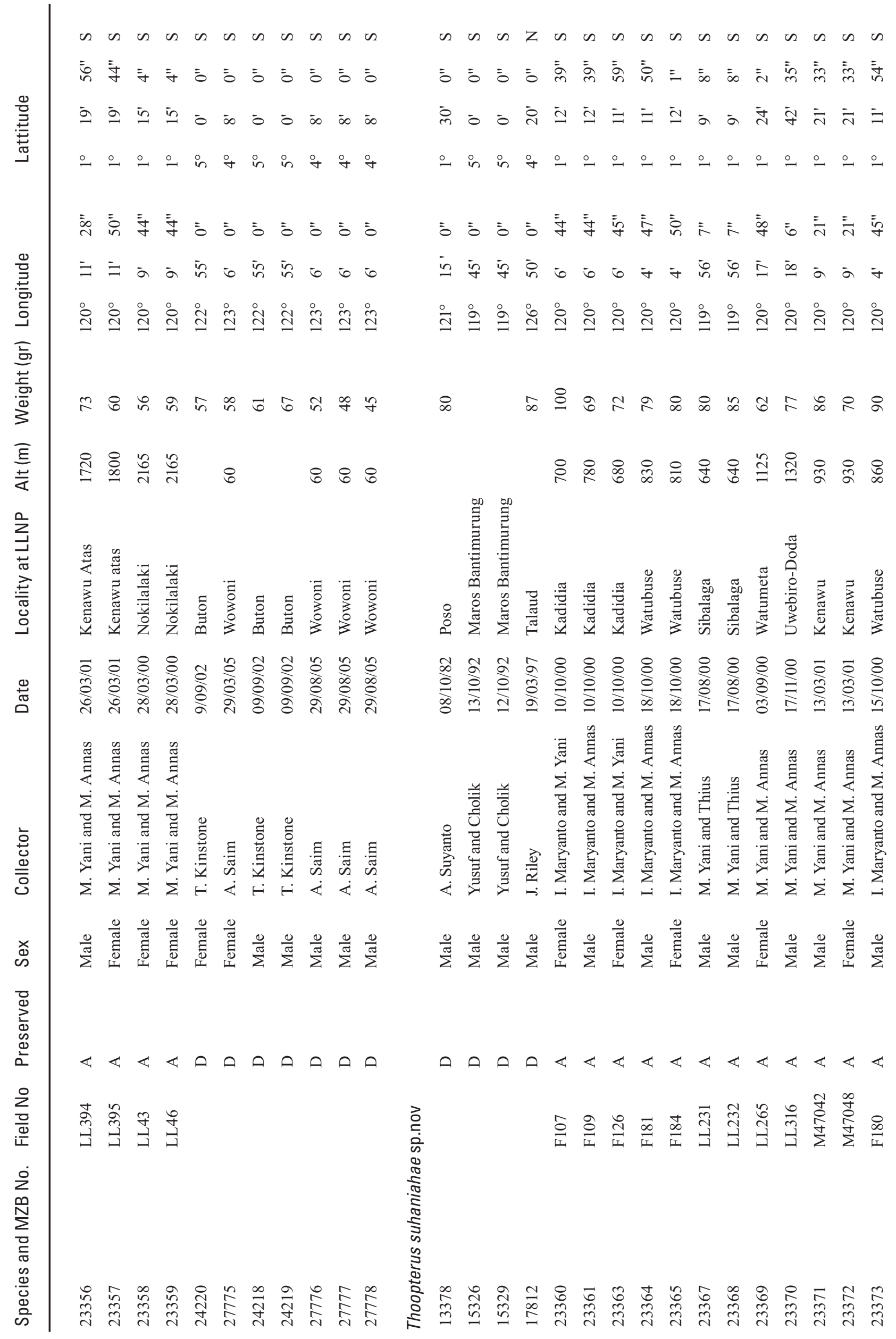




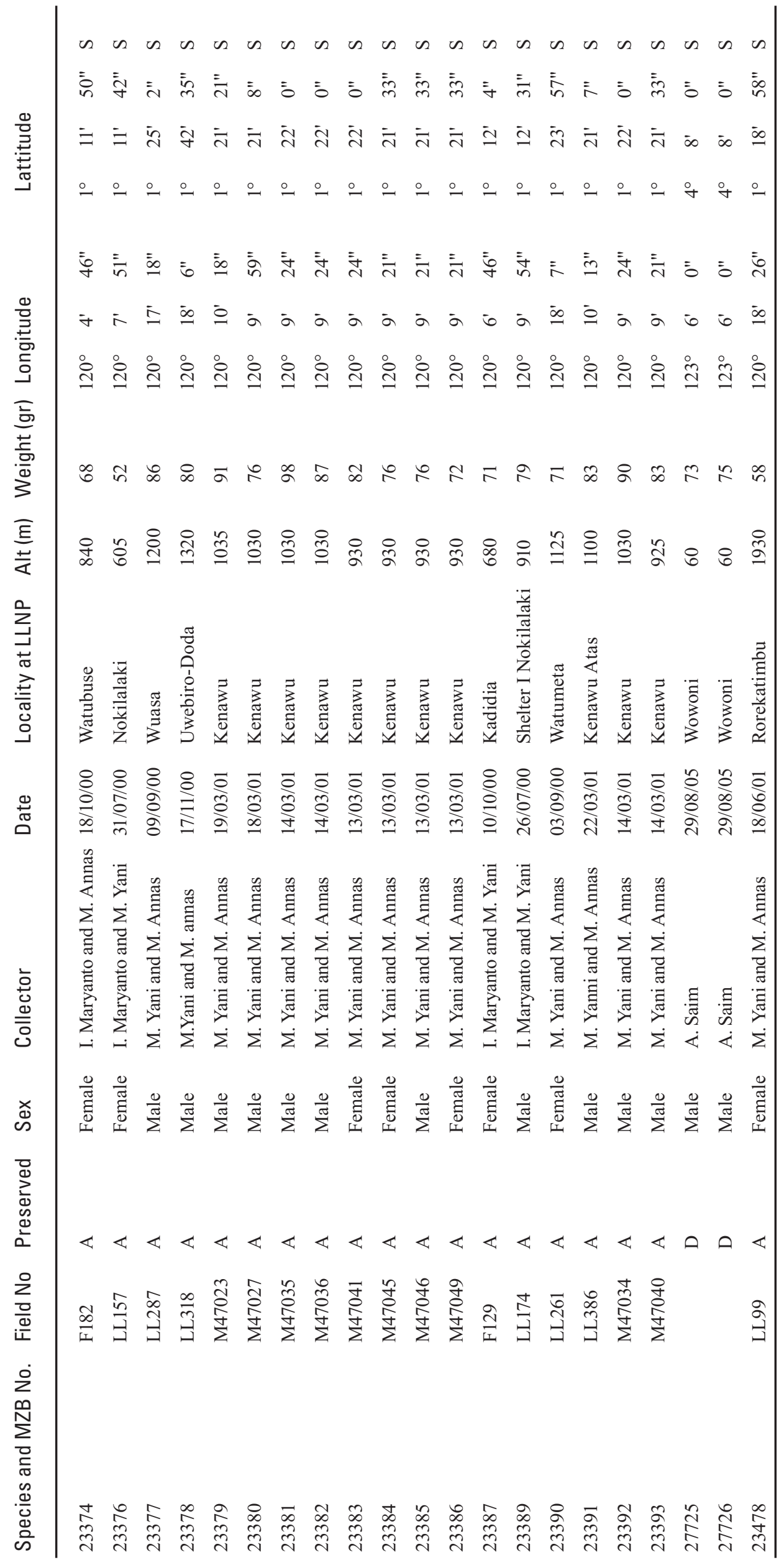

